\title{
Análisis del discurso publicitario de las academias preuniversitarias en las regiones Cusco y Ayacucho
}

\section{Analysis of the advertising discourse of pre-university academies in the Cusco and Ayacucho regions}

\author{
Ivonne Alessandra Paredes Ramírez \\ Universidad Nacional Mayor de San Marcos, Lima, Perú \\ ivonne.paredes@unmsm.edu.pe
}

\begin{abstract}
Resumen
Para muchos egresados de la educación secundaria, las academias preuniversitarias son el camino necesario que se debe tomar para poder asegurar una vacante en la universidad. El propósito de este trabajo es identificar y describir las estrategias discursivas que estas academias manejan mediante los avisos publicitarios que muestran en la red social Facebook para así demostrar que las academias convencen y persuaden mediante su discurso a los estudiantes.
\end{abstract}

Palabras clave: Academias preuniversitarias; discurso; avisos publicitarios; red social; estrategias discursivas.

\begin{abstract}
For many high school graduates, pre-university academies are the necessary path to take to secure a college vacancy. The purpose of this work is to analyze the discourse that these academies handle through the advertisements they show on the social network Facebook, as well as to make a description of the discursive strategies present in these advertisements.

Keywords: Pre-university academies; speech; advertisements; social network; discursive strategies.
\end{abstract}


Análisis del discurso publicitario de las academias preuniversitarias en las regiones... Ivonne Alessandra Paredes Ramírez

\section{Introducción}

La etapa preuniversitaria en el Perú es un proceso lleno de mucha tensión y ansiedad para los adolescentes. Cuando acaba la etapa escolar, la mayoría decide estudiar una carrera universitaria; pero se sabe que la educación en la escuela no es suficiente para poder ingresar a la universidad. Por ello, se hace necesario estar en alguna academia para completar los conocimientos básicos y de esa manera lograr obtener una vacante en algún centro de estudio superior.

Es ahí cuando el mercado de las academias surge y en la actualidad es un boom. Actualmente, hay cada vez más academias, de todos los precios, de todos los tipos, ofreciendo infinidad de cosas, desde lo más común (como un local en el que haya salones y se dicten clases) hasta clases a domicilio, de manera virtual, etc.

No hay trabajos anteriores que traten sobre el mismo tema (anuncios preuniversitarios). El antecedente más cercano es la tesis de María Sanabria (2016), donde realiza un análisis pragmático-textual de los anuncios publicitarios de productos de belleza. El objetivo de esta investigación es demostrar cómo es que el discurso publicitario de las academias peruanas busca persuadir a que el estudiante se matricule en ellas y este crea que solo de esa manera podrá ingresar a la universidad.

\section{Marco Teórico}

\subsection{Análisis del discurso}

Fairclough (1992) concibe el término «discurso» como una práctica social, la cual se enuncia como el accionar de individuos concretos. Este accionar expresa creencias sobre sí mismos y los demás (Foucault, 1999; Lovón, 2018), por lo que se puede decir que el discurso involucre pensamientos, opiniones y relaciones de poder que le permite a los individuos posicionarse en el espacio social (Hayakawa, 1967).

Además, Fairclough asevera que el discurso es un modo de acción y de representación, donde existe una relación dialéctica entre discurso y estructura social. Cabe indicar que la práctica social se encuentra dentro de la estructura social. Para Fairclough, el discurso es una práctica que constituye y construye a partir del significado.

$50 \quad$ Lengua \& Sociedad. Revista de Lingüística Teórica y Aplicada 


\subsection{Estrategias discursivas}

Las estrategias discursivas, según Fairclough (2008), pueden ser los recursos semántico-gramaticales (asignación de roles semánticos en las oraciones), vocabulario (léxico que se usa para referirse a alguna entidad), la organización dialógica (citado de voces ajenas en una conversación), la cohesión entre las cláusulas en oraciones complejas.

Asimismo, Fairclough señala que estas estrategias son necesarias para la producción e interpretación del contenido. Este análisis interpretativo está relacionado con la práctica discursiva (Lovón, 2019). A su vez, esta se configura así a partir de los aspectos sociocognitivos. Fairclough (1992) indica que estos aspectos refieren a los recursos que el usuario del lenguaje ha internalizado y que usa para interpretar y producir textos.

\section{3 Éxito universitario}

Arthur et al. (2005) citado por Caicedo (2007) dan un albor de qué significado tiene el éxito, para ello proponen dos conceptos de éxito a partir de dos acepciones de éxito del Diccionario Oxford:

a. «el logro de un objeto de acuerdo con nuestro deseo», que sugiere un enfoque personal (éxito subjetivo)

b. «el logro próspero de algo que se ha intentado», que implica una forma de éxito que depende de las comparaciones sociales (éxito objetivo).

Según el DLE la palabra éxito presenta 3 acepciones a) Resultado feliz de un negocio, actuación, etc., b) Buena aceptación que tiene alguien o algo y c) Fin o terminación de un negocio o asunto. Para la palabra universitario la definición que se da es la siguiente: Profesor, graduado o estudiante de universidad. En ese sentido se podría afirmar que el éxito universitario es el feliz resultado de culminar la etapa universitaria.

Fernández y Cantón (2017) concluyen en que el éxito se asocia, en el caso académico, al esfuerzo y los resultados y que se correlaciona con variables como el género, el bienestar psicológico, la resiliencia o el uso de las tecnologías.

\subsection{Publicidad}

La publicidad es una forma de comunicación que pretende dar a conocer a través de mensajes publicitarios, productos, servicios, ideas o instituciones con los 
Análisis del discurso publicitario de las academias preuniversitarias en las regiones... Ivonne Alessandra Paredes Ramírez

objetivos fundamentales de informar sobre ellos o influir en su compra (Ferraz, 1995; Fernández, Flores y Lovón, 2018).

Sanabria (2016) menciona que en la publicidad hay dos tipos de objetivos según Thompson: los generales y los específicos.

Como objetivos generales se tienen a informar, persuadir y recordar tal y como lo propone Kotler (2012). Stanton, Etzel y Walker (2007), menciona Sanabria, proponen los siguientes objetivos específicos: dar respaldo a las ventas personales, mejorar las relaciones con los distribuidores, introducir un producto nuevo, expandir el uso de un producto y contrarrestar la sustitución.

\section{Metodología}

Este trabajo es de carácter descriptivo y de tipo cualitativo. Para conseguir la muestra primero se buscaron en internet qué academias había en Cuzco y Ayacucho. Después de seleccionar los nombres, se prosiguió con la búsqueda de sus respectivas páginas de Facebook. Posteriormente, se descargaron los avisos publicitarios que contenían la mayor cantidad de información en ellos. Fueron 4 las academias analizadas y el recojo de la data se dio el día 11 de octubre del 2019. Se obtuvo un total de 17 eslóganes o frases y se clasificaron de la siguiente manera:

- Mensajes de éxito

- El éxito como resultado del esfuerzo

- Comprometidos con el éxito

- Resultados del éxito

- Liderazgo

- Asegurar ingreso a la universidad

- Característica propia

- Beneficios

- Frases motivadoras

Cabe resaltar que, en cuanto a la ortografía, se han transcrito los eslóganes tal cual se muestra en las imágenes; es decir, respetando el uso de mayúsculas y minúsculas y colocando los signos de puntuación. 
Análisis del discurso publicitario de las academias preuniversitarias en las regiones... Ivonne Alessandra Paredes Ramírez

\subsection{Corpus}

\section{Academia Raimondi}

(a) El éxito en la vida no se mide por lo que logras, sino por los obstáculos que superas.

(b) Juntos: Raimondi y Tú COMPROMETIDOs para LOGRAR el ÉXITO

(c) EL SACRIFICIO DE HOY, ES EL ÉXITO DE MAÑANA.

(d) Siempre los primeros, dejando huella

(e) BONO DE DESCUENTO S/.50

\section{Academia Pardo}

(f) ROTUNDO ÉXITO DE LA ACADEMIA PARDO EN EL EXAMEN ORDINARIO 2015-I

(g) 7 ingresantes a ING. CIVIL obteniendo la nota más alta en el cómputo general

(h) VEN, PREPÁRATE CON NOSOTROS Y ASEGURA TU INGRESO

(i) ...! El poder del conocimiento!!!

(j) EXAMEN DE BECAS Gratis!

\section{Academia Lobachevski}

(k) Si vas a la UnSCH Estás en el camino

(l) INGRESE A LA UNIVERSIDAD

\section{Academia Falcons}

(m) EL ÉXITO es la suma de GRANDES ESFUERzos que se repiten CADA DÍA Tranquilo, en LOBACHEvski lo hacemos fácil y seguro tu ingreso.

(n) LA MEJOR ACADEMIA DE AYACUCHO

(o) Calidad y Exigencia

(p) SEMINARIOS INVERSIÓN 4-8PM S/. 3.00

(q) «RODÉATE DE GENTE CON EL MISMO OBJETIVO QUE TÚ» 
Análisis del discurso publicitario de las academias preuniversitarias en las regiones... Ivonne Alessandra Paredes Ramírez

\section{Análisis de los datos}

\subsection{Los mensajes de éxito}

En las cuatro academias analizadas se encuentran mensajes de éxito. Ello con la consigna de poder motivar a adolescentes y jóvenes tanto de la región Cusco como de la región Ayacucho a que ingresen a la universidad. Se hallaron seis mensajes de texto y se clasificaron de la siguiente manera: el éxito como resultado del esfuerzo (a, c, k), comprometidos con el éxito (b) y resultados del éxito (f, m).

En (a) observamos que se trata de una oración adversativa, puesto que se encuentra el nexo sino, el cual denota que hay ideas contrapuestas en torno a cómo es que se obtiene el éxito. Se da prioridad y mayor importancia al proceso por el que se tiene que pasar (superar obstáculos) para conseguir algo que el mismo hecho de haberlo conseguido (el éxito en la vida no se mide por lo que logras). El éxito, para la academia Raimondi, claramente es el ingreso a alguna universidad. La selección léxica de esta oración es la siguiente: éxito, mide, lograr, obstáculos y superar.

La oración (c) nos muestra la misma idea con la ligera diferencia de que en esta oración se afirma que debe haber un sacrificio (un acto de abnegación inspirado por la vehemencia del amor según la acepción 7 del $D L E$ ) para que se dé el éxito, sin ese sacrificio no habrá éxito, sacrificarse se torna una condición necesaria para ser exitoso. También se puede observar el uso de adverbios temporales hoy y mañana para indicar el imperativo de tener que realizar el sacrificio. Por último, el uso de verbo ser en tiempo presente tiene el fin de equiparar ambas premisas (sacrificio de hoy = éxito de mañana).

La diferencia de (k) en relación con las oraciones (a) y (c) es que en esta tercera no se menciona de manera explícita al éxito. Se trata, pues, de una oración condicional y esto se evidencia con la presencia del nexo si; es decir, si quieres ingresar a la UNSCH tienes que prepararte en la academia Lobachevski porque solo así conseguirán alcanzar una vacante. Cabe resaltar que el hecho de mencionar específicamente a la UNSCH indica que se está colocando a la Universidad Nacional San Cristóbal de Huamanga como el rol meta o el destino.

En (b) el discurso de la academia cambia, ya no es el estudiante quien tiene que hacer sacrificios para conseguir el éxito; o sea, ingresar, sino que ahora la academia se compromete con el alumno para así juntos poder lograr el objetivo, 
Análisis del discurso publicitario de las academias preuniversitarias en las regiones...

Ivonne Alessandra Paredes Ramírez

creando así una especie de lazo de confianza para conseguir la mayor cantidad de alumnado. La estructura es la siguiente:

Juntos: Raimondi y Tú COMPROMETIDOS para LOGRAR el ÉXITO (b)

Como se puede observar, se usa el término juntos para hacer hincapié en que el alumno no está solo, que es un trabajo de dos y que solo así se podrá alcanzar el objetivo. El propósito de colocar palabras en mayúsculas es resaltarlas porque son el tema central: COMPROMISO (de las dos partes) = INGRESAR a la universidad. También se observa el tuteo que es una de las formas de tratamiento de confianza del español. Algo particular a nivel de la ortografía es el uso de la mayúscula para la consonante de la palabra tú y esto con el fin de resaltar la presencia del estudiante. A continuación, la selección léxica en esta oración: juntos, comprometidos, lograr y éxito.

La siguiente frase es la oración (f), en ella ya no se verá el motivo éxito ni como un compromiso; sino los resultados del éxito:

\section{ROTUNDO ÉXITO DE LA ACADEMIA PARDO EN EL EXAMEN ORDINARIO 2015-I}

Claramente se nota que el éxito es que algunos o quizá muchos alumnos de la academia Pardo hayan ingresado a la universidad en el examen ordinario 2015-I. La palabra rotundo según su definición en el Diccionario de la lengua española (DLE) en la acepción 3 nos dice \|Completo, preciso y terminante. Esto quiere decir que no hubo motivo a falla y que, efectivamente, los estudiantes de la academia Pardo lograron ingresar a la universidad.

Por último, la frase $(\mathrm{m})$ refleja el resultado del sacrificio y la perseverancia: el éxito. Poder ingresar a la universidad es un hecho que no solo está ligado al conocimiento de las materias; sino también a la constancia y al esfuerzo. Y, claro, no se debe hacer sacrificios de manera esporádica, la constancia es de todos los días, tal y como lo menciona la academia Falcons. En la oración se halla la presencia de una subordinación específica (que se repiten) que está presente con el fin de hacer hincapié en que los esfuerzos deben repetirse constantemente y no es ajeno que después de la subordinación se encuentre el adverbio temporal cada junto al nombre día para realzar aún más el tener que sacrificarse siempre. 
Análisis del discurso publicitario de las academias preuniversitarias en las regiones... Ivonne Alessandra Paredes Ramírez

\subsection{El liderazgo académico}

En el corpus también se hallaron mensajes sobre liderazgo, tres de las cuatro academias analizadas usan estos tipos de mensajes en su publicidad con la finalidad de hacerle conocer al estudiante que son las mejores y que eso es una característica necesaria como garantía para que puedan alcanzar una vacante en la universidad.

La oración (d) se caracteriza por usar el adverbio polarizador siempre y la metáfora huella para hacer referencia a que la academia Raimondi siempre figura dentro de las academias que logra el que más alumnos ingresen a la universidad.

En la oración (g) la estructura es la siguiente:

7 ingresantes a ING. CIVIL obteniendo la nota más alta en el cómputo general

Notamos que se hace uso de números o cifras para aumentar el prestigio de la academia, no se trata solo de que 7 personas ingresen a estudiar la carrera de Ingeniería Civil, sino que son 7 alumnos que han tenido la NOTA MÁs ALTA. Esto último refleja que obtener la nota más alta es lo mejor. Por otro lado, al mencionar el nombre de la carrera se hace un destaque de especial; es decir, se menciona esta carrera porque se sabe que todas las ingenierías son profesiones que son muy bien remuneradas y que tiene prestigio en el ámbito laboral, no se observa que se hable sobre los ingresantes a las carreras del área de Humanidades, por ejemplo. Dentro de la selección léxica tenemos cómputo general y se usa esta frase nominal como una fuente para demostrar la veracidad de lo que se dice.

Por último, en la oración (n) la academia Falcons hace uso del adjetivo mejor. El DLE define a esta palabra en sus tres primeras acepciones de la siguiente forma:

1. Superior a otra cosa y que la excede en una cualidad natural o moral.

2. Preferible o más conveniente.

3. Más bien, de manera más conforme a lo bueno o lo conveniente.

Esto denota que la academia Falcons refiere ser la mejor y no solo de la zona, sino de toda la región de Ayacucho. Al mencionar el nombre de la región se busca obtener la identidad o la pertenencia de la academia.

\subsection{Asegurar ingreso a la universidad}

Para esta sección solo las academias Pardo y Lobachevski son las que le aseguran al estudiante alcanzar una vacante en la universidad; es decir, no hay margen de error, el alumno ingresará.

La oración (h) tiene la siguiente estructura:

$56 \quad$ Lengua \& Sociedad. Revista de Lingüística Teórica y Aplicada 
Análisis del discurso publicitario de las academias preuniversitarias en las regiones... Ivonne Alessandra Paredes Ramírez

VEN, PREPÁrATE CON NOSOTROS Y ASEGURA TU INGRESO

Primero se busca apelar cuando se coloca el verbo venir en modo de orden o mandato. También está el uso del pronombre nosotros para indicar que la academia Pardo es un conjunto de individuos que formarán parte del logro; es decir, el alumno no estará solo, muy por el contrario, habrá muchas personas encargadas de su avance académico. Como parte de la selección léxica se encuentra la palabra asegura la cual significa según el DLE: Confirmar o aseverar la realidad o certeza de algo (acepción 3). Y, para finalizar con el análisis de esta oración está el tuteo que también ha sido mencionado párrafos arriba. Sin embargo, ahora no solo está en la forma del pronombre de segunda persona (tú), sino que también se encuentra como pronombre enclítico (te).

En la oración (l) también hay tuteo (con la partícula $t u$ ) pero también se manifiesta la formalidad en la primera palabra (ingrese), puesto que no se trata de que tú ingreses, sino de que usted ingrese. Nuevamente existe el tema de colocar el nombre de la academia (Lobachevski) como membresía para resaltar el nombre de la entidad. En cuanto a la selección léxica, se usa el término fácil que está definido como que no requiere gran esfuerzo, habilidad o capacidad, según el DLE.

\subsection{Marca de la academia}

Se entiende como característica propia a la marca que o referencia que solo se hace a una academia. Por ejemplo, en (i) y (o) tan el poder del conocimiento como calidad y exigencia son características o 'segundos nombres' tanto de la academia Pardo como de la academia Falcons.

La selección léxica de estas frases son las siguientes palabras que serán definidas según el DLE:

- Poder: Tener expedita la facultad o potencia de hacer algo.

- Conocimiento: Entendimiento, inteligencia, razón natural.

- Calidad: Propiedad o conjunto de propiedades inherentes a algo, que permiten juzgar su valor.

- Exigencia: Pedir imperiosamente algo a lo que se tiene derecho.

\subsection{Beneficios}

En cuanto a los beneficios, (e), (j) y (p) serán ayudas que las academias brindarán para que los estudiantes puedan tener mayor facilidad al momento de querer 
Análisis del discurso publicitario de las academias preuniversitarias en las regiones... Ivonne Alessandra Paredes Ramírez

iniciar una preparación y así postular a la universidad. Estas facilidades refieren al dinero, en el primer caso se hará un descuento de 50 soles al total del monto a pagar. En el segundo caso se resalta la gratuidad para dar un examen y asó obtener el poder estudiar sin hacer pago alguno en la academia Pardo. Y, en el último caso se da un precio mínimo por llevar seminarios que ayudarán a reforzar los conocimientos que el alumno posea en las clases. Las academias hacen todo ello para ayudar, en este caso de manera económica, a la población que menos recursos económicos tiene.

\subsection{Frases motivadoras}

Solo la academia Falcons hace uso de una frase motivadora, en ella se observa nuevamente el fenómeno del tuteo tanto en pronombre enclítico (rodéate) como en el uso del pronombre persona de segunda persona (tú). Y, pues, en sí la frase hace referencia a que el estudiante debe interiorizar muy bien el dicho 'dime con quién andas y te diré hasta donde puedes llegar.'

Para asegurar mayor éxito se necesita buscar personas que estudien mucho más que otras o que demuestren (en los hechos) tener como verdadera prioridad el ingresar a la universidad y a esas personas se las debe colocar en el círculo interno del estudiante. El alumno no debería invertir todo su esfuerzo en convencer o inspirar a personas negativas. Por el contrario, debe invertir su energía en las personas que comparten su visión.

\section{Conclusión}

Se ha demostrado cómo es que el discurso publicitario de las academias preuniversitarias tanto de la región Cusco como de la región Ayacucho buscan obtener la mayor cantidad de estudiantes usando la técnica de hablar bien de sí mismos y de dar a conocer los logros que en el devenir del tiempo han obtenido. Todas las academias afirman que es posible ingresar a la universidad sí y solo sí el alumno se prepara en ellas y no de otra forma.

Un fenómeno recurrente y que manejan la mayoría de las academias es el tuteo y la selección léxica; es decir, el uso de determinadas palabras que le dan más valor o relevancia a lo que se quiere decir.

58 Lengua \& Sociedad. Revista de Lingüística Teórica y Aplicada 


\section{Referencias Bibliográficas}

Fairclough, N. (2008). El análisis crítico del discurso y la mercantilización del discurso público: las universidades. Discurso y Sociedad, 2(1), 170-185.

Fairclough, N. (1992). Discourse and social change. Cambridge: Polity.

Fernández, C., Flores, C. y Lovón, M. (2018). Análisis metafórico de los anuncios publicitarios relacionado con la mujer como objeto sexual. Lengua \& Sociedad, 17(1), 100-200. Recuperado de http://revista.letras.unmsm.edu.pe/index.php/ 1s/issue/view/48

Ferraz, A. (1995). El lenguaje de la publicidad. Madrid: Arco/Libros.

Foucault, M. (1999 [1966]). Las Palabras y las Cosas. Madrid: Siglo XXI.

Hayakawa, S. I. (1967). El lenguaje en el pensamiento y en la acción. México: Uteha. Lovón, M. (2019). El «ciudadano» amazónico en el discurso oficial. Lingüística y Literatura, 75, 38-61. https://doi.org/10.17533/udea.lyl.n75ao2

Lovón, M. (2018). Análisis crítico del discurso lexicográfico (ACDL): un examen a la historia de las lenguas andinas en los diccionarios de la RAE. Boletín de la Academia Peruana de la Lengua, 64(64), 141-171. Recuperado de http://revistas. academiaperuanadelalengua.org.pe/index.php/boletinapl/article/view/181

Real Academia Española. (2014). Diccionario de la Lengua Española. España: Espasa.

Sanabria, M. (2016). Análisis pragmático-textual de los anuncios publicitarios de productos de belleza (Tesis de licenciatura). Universidad Nacional Mayor de San Marcos, Lima. 
Análisis del discurso publicitario de las academias preuniversitarias en las regiones... Ivonne Alessandra Paredes Ramírez

\section{Anexos}

Academia Raimondi (Cusco)

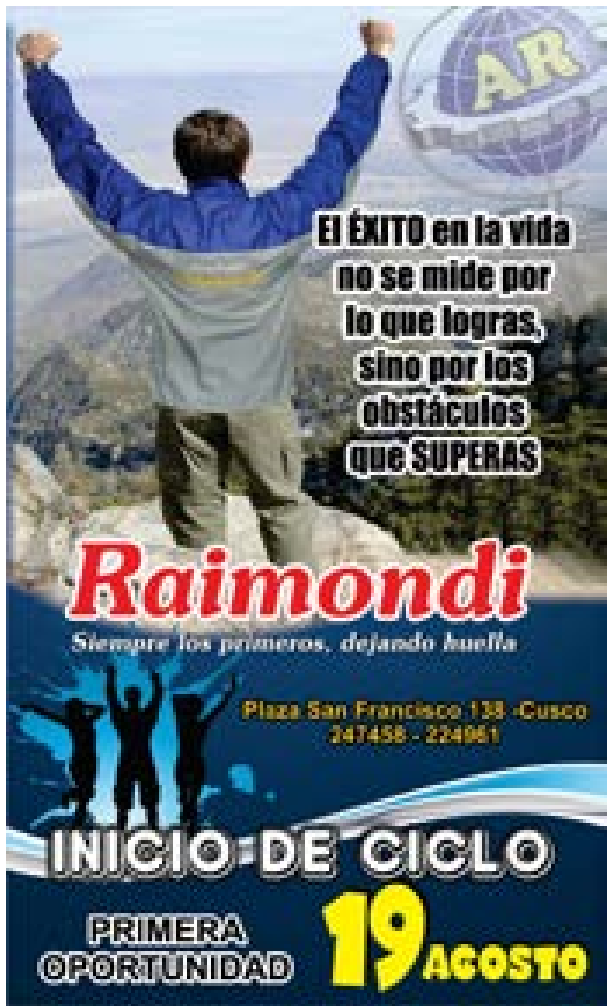

18 de julio del 2019

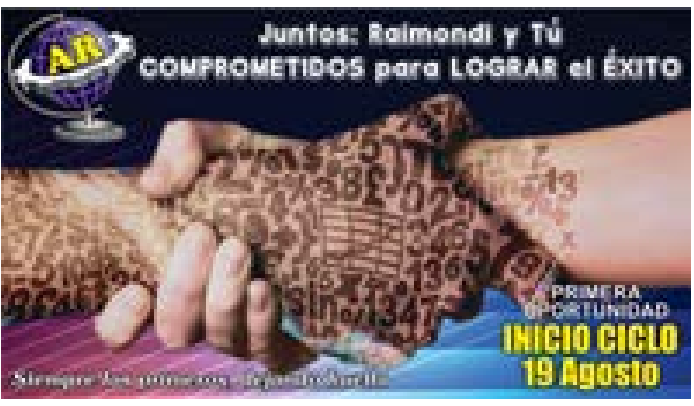

18 de agosto del 2019

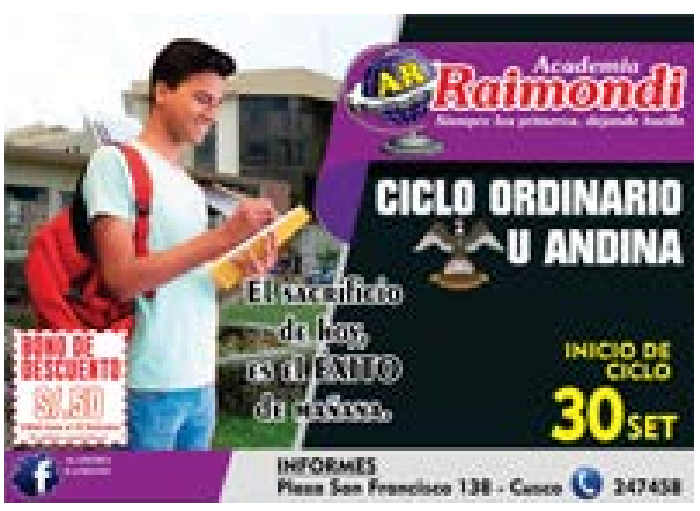

12 de setiembre del 2019 
Análisis del discurso publicitario de las academias preuniversitarias en las regiones... Ivonne Alessandra Paredes Ramírez

\author{
Academia Pardo (Cusco)
}

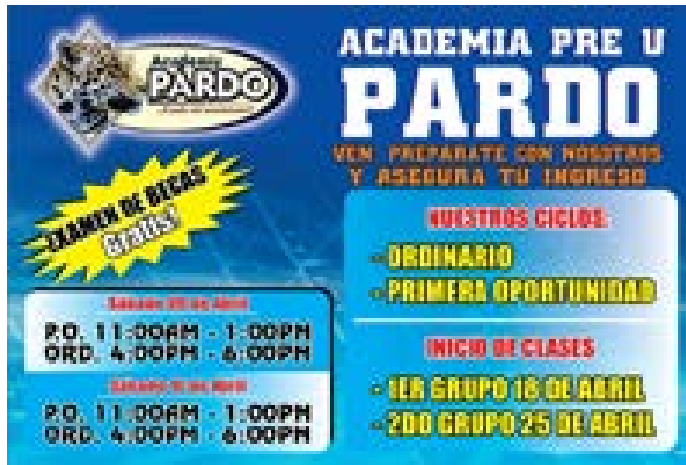

28 de marzo del 2019

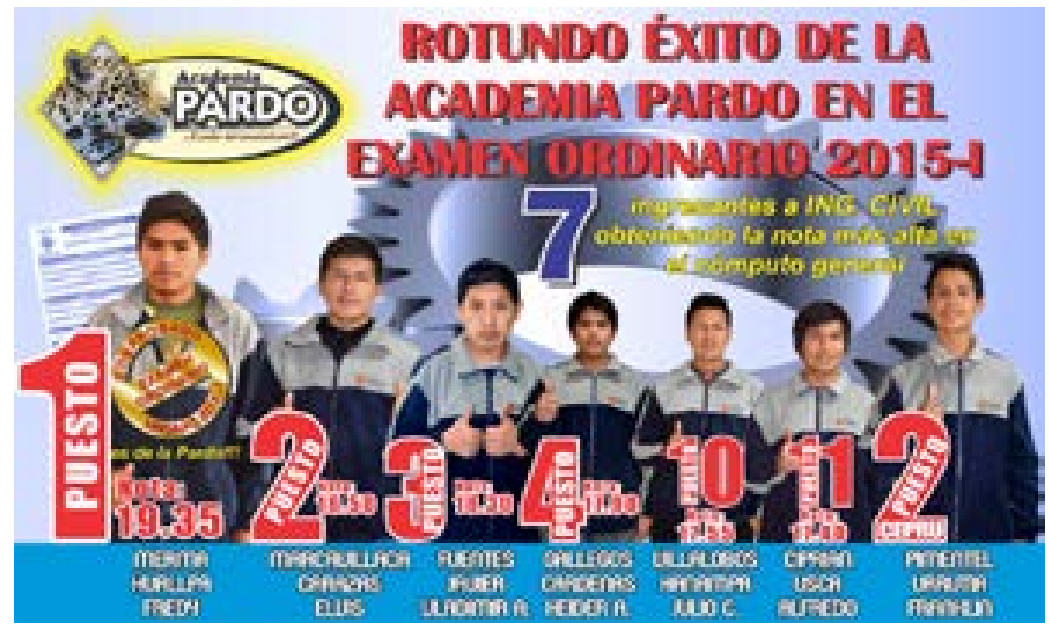

28 de marzo del 2015 
Análisis del discurso publicitario de las academias preuniversitarias en las regiones... Ivonne Alessandra Paredes Ramírez

Academia Lobachevski (Ayacucho)
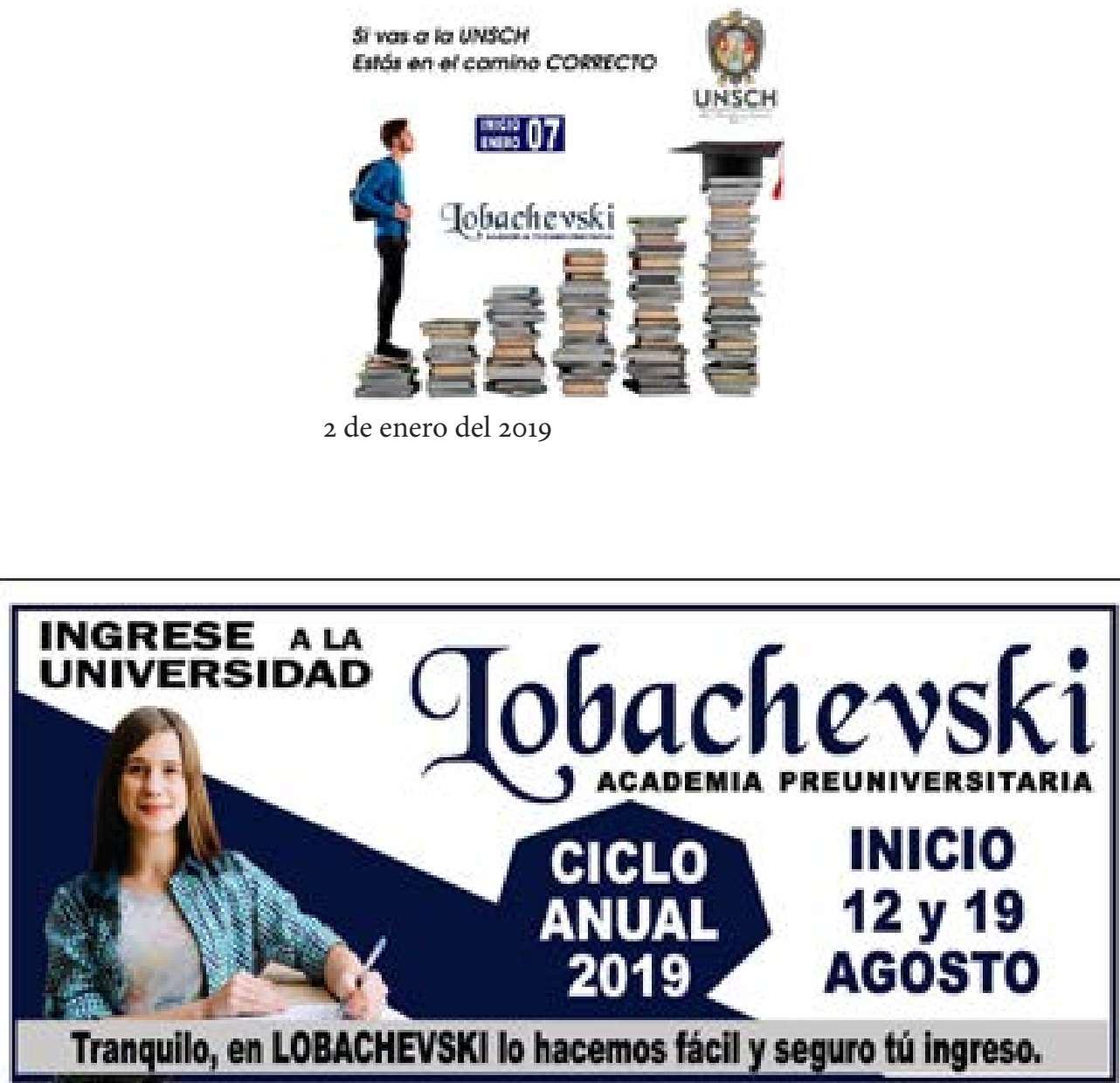

2 de enero del 2019 
Análisis del discurso publicitario de las academias preuniversitarias en las regiones... Ivonne Alessandra Paredes Ramírez

\section{Academia Falcons (Ayacucho)}

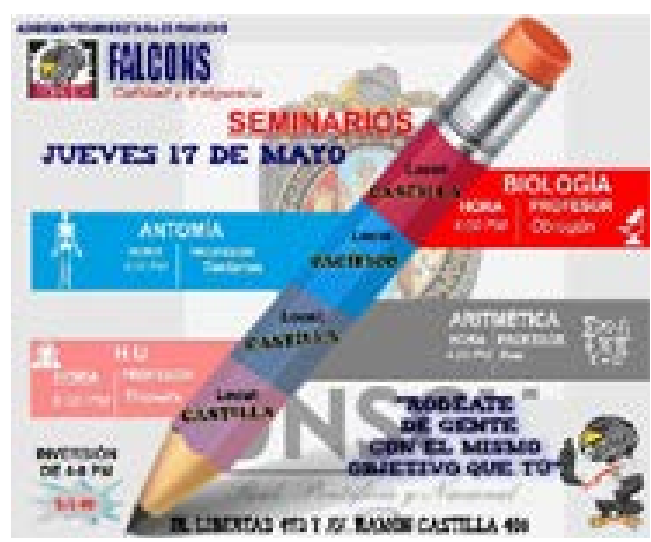

17 de mayo del 2018
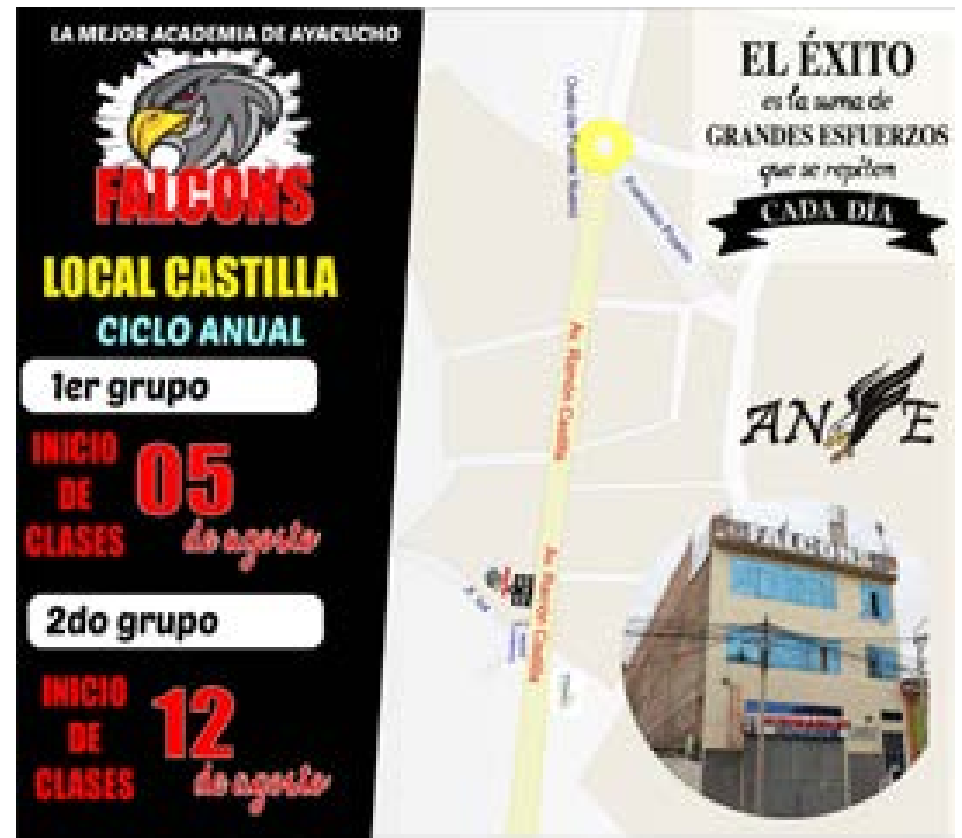

4 de agosto del 2019 